\title{
Outpatient screening endoscopy: Why not the standard of care?
}

\author{
Michael Gould MD¹, Paul Adams MD², Editor-in-Chief
}

$D^{\prime}$ $r$ Michael Gould is a community gastroenterologist in Toronto, Ontario. He is a Partner and President of Vaughan Endoscopy Clinic Inc (Vaughan, Ontario). He currently serves on the Board of Directors of the Ontario Association of Gastroenterology (and is a past President) and the Canadian Digestive Health Foundation. He served on many Cancer Care Ontario (CCO) committees dating back to 1999 , and was a member of the College of Physicians and Surgeons of Ontario's (CPSO)

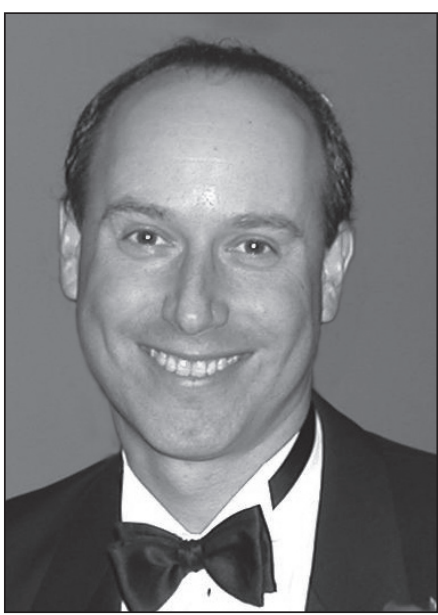

Dr Michael Gould is a community gastroenterologist in Toronto, Ontario. He is a Partner and President of Vaughan Endoscopy Clinic Inc, Vaughan, Ontario

offices, cardiology offices and often in hospital endoscopy units, quality assurance is the responsibility of the doctors performing the procedures. While the vast majority of physicians are wellintentioned and do their best to protect their patient's interests, there are few clear standards to follow, and those that do exist often change frequently and without the knowledge of the endoscopists. Some examples of evolving standards are infection control and privacy issues. It would appear that there are a few clinics or groups

Independent Health

Facility (IHF) Endoscopy Committee. He is the past Chief of Medicine of the William Osler Health Centre in the greater Toronto area and Vice President of the Toronto Digestive Disease Associates Inc Clinical Gastroenterology Research Group.

PA: As waiting lists for colonoscopy continue to grow in Ontario, we are seeing ambulatory endoscopy clinics starting in many urban areas. These clinics may be owned by a business partner or the doctors. Some charge facility fees and most are paying the overhead out of professional fees. A major concern of the government has been the assurance of quality control at these endoscopy centres. Who is in charge of quality control?

MG: As in all aspects of medicine, including clinical gastroenterology that try to cut corners intentionally. Unfortunately, there are no organizations that are empowered to ensure quality endoscopy in the outpatient setting. It would appear, for political reasons, that no agency has been created nor organization charged with accrediting endoscopy clinics in Ontario. The CPSO has created IHF guidelines. CCO has created quality of care guidelines and the Canadian Association of Gastroenterology has published screening recommendations (1). These three documents could make up the constituent elements of an endoscopy facility accreditation program. The politics are such that, despite the fact that the ideal venue to perform screening colonoscopies on our healthiest patients would be ambulatory clinics and not our overburdened hospitals, there is active

\footnotetext{
${ }^{1}$ Toronto Digestive Disease Associates Inc, Toronto; ${ }^{2}$ University Hospital, London, Ontario

Correspondence: Dr Michael Gould, 89 Humber College Boulevard, Suite 222, Etobicoke,

Ontario M9V 4B9.

Telephone 416-745-9994, fax 416-745-3653, e-mail mgouldgi@rogers.com

Received and accepted for publication December 5, 2008
} 
avoidance by the government and $\mathrm{CCO}$ to endorse the more efficient out-of-hospital endoscopy clinic model to deliver needed screening services to the healthy screening population. Because there is no formal acceptance of outpatient facilities, there is no place for any organizations or groups to assume responsibility for accrediting those facilities. There is also a reluctance to provide the necessary and appropriate funding. The acknowledgement (by the Ontario government and CCO) that these accredited facilities would be the ideal place for the delivery of endoscopic services (to the healthy screening population) would necessitate the requirement for adequate funding (the clinics provide the infrastructure at a lower cost than the hospitals and deliver the services more efficiently). The vacuum created by not acknowledging the need for, or the existence of, appropriate endoscopy facilities results in questions regarding quality of care and resource use.

PA: You hear a lot of rumours about the standard of care at some of these centres. Can you outline for us the best and worst of scenarios that may be occurring in the Toronto area?

MG: Most of what you hear is rumour, but with the reimbursement coming primarily from professional fees and not technical fees, the rumours suggest there may be issues with undersedation, old (ie, not current) equipment, poor monitoring equipment, understaffing or the use of staff without accepted credentials. There are clinics that employ less-experienced endoscopists. These endoscopists may have completion rates below acceptable standards, not follow withdrawal time recommendations and may not be experienced with more complicated polyp removals. In addition, because they are not specialists in gastrointestinal disease, they do not provide the necessary postendoscopy follow-up for screening patients who have have gastrointestinal symptoms that may require further workup or follow-up. There are a number of different endoscopy clinic models that exist in the greater Toronto area and some are clearly better than others. We need to develop a process to ensure that all outpatient endoscopy is performed safely and to a high standard.

PA: Why have we not seen a technical fee for colonoscopy when other specialties (eg, echocardiography, ultrasound) have fees to support their services?

MG: Again, this is a political issue. There are those who believe that because radiology and cardiology have technical fees, the doctors are remunerated excessively. What has been forgotten, for the most part, is that the tests are being ordered by other physicians in an effort to care for their patients. Often, the patients demand the services. If the services were not offered in the ambulatory setting or outpatient setting, the burden of the recommended tests would fall on the hospitals or not be provided at all. Because the vast majority of doctors are trying to do their best for the patients, the majority of tests (even the standard ones) are required for the investigation of complaints. I believe that the failure to provide adequate funding is very shortsighted, particularly as the demand for screening increases. I believe that colon cancer screening is now an acceptable indication for colonoscopy throughout North America and Europe. Because colonoscopy is a medically necessary procedure, increasing demand will require an increasing colonoscopy capacity. Technical fees are not the only funding model, and I am not sure if technical fees are the best model, but I am sure that funding for the technical component of outpatient endoscopy is critical to ensure that we increase the availability of the required high-quality colonoscopy services.

PA: Who is qualified to be performing colonoscopy at ambulatory centres?

MG: Different training programs produce endoscopists with different skill sets. The Royal College of Physicians and Surgeons of Canada requirements for endoscopic proficiency are different among specialty groups. Not all endoscopists maintain adequate continuing medical education hours in endoscopy. Simply said, endoscopists must be properly trained and experienced. Well-trained endoscopists have 98\% cecal intubation rates, understand and perform proper withdrawal times, recognize the existing pathology, are able to perform the required therapeutics for polyp removal and can expertly deal with rare but inevitable complications. They should also be skilled in managing the patient's gastrointestinal issues. Endoscopy is not just another test but part of the comprehensive care program that gastroenterologists provide to their patients. The willingness to follow up patients is vital. The clinics should not resemble laboratories or diagnostic radiology clinics. It is not just about performing colonoscopy - the care provided should include the procedure, but also its interpretation, treatment and follow-up of the findings.

PA: Do you foresee complementary platforms such as virtual colonoscopy or DNA testing existing in a modern ambulatory endoscopy centre?

MG: There are many ways to screen patients. Screening with any technique is better than no screening at all. I am not sure of the role of virtual colonoscopy for screening in our province or country. We have a shortage of computed tomography (CT) scanners and, in particular, of the new multislice scanners that deliver a reduced radiation dose. I believe that the lifetime cumulative radiation exposure from virtual screening programs that use older scanners (ie, less than 16-slice) is unacceptable. Furthermore, if you think it is important to remove polyps larger than $5 \mathrm{~mm}$ to $6 \mathrm{~mm}$ in size, then up to $30 \%$ or more of screened patients will require a subsequent colonoscopy. The current wait time for CT is substantial without screening. Without increasing CT capacity, the introduction of screening by virtual colonoscopy will prolong wait times unacceptably. If we are going to spend money to increase CT capacity, we could clearly increase funding for outpatient colonscopy. If we can solve some of the CT issues, we may consider combining the modalities into a single screening centre as a more efficient way to provide screening.

PA: What are the greatest impediments to the growth of ambulatory endoscopy centres in Canada?

MG: The growth in the number of clinics has been significant despite inadequate funding. The unfortunate outcome is the absence of controls with respect to quality and expertise. Often driven by costs or third-party financial interests, there are a number of clinics whose equipment, processes or staffing may be less than ideal. Within the community, there is concern over this, and has resulted in groups such as $\mathrm{CCO}$ being reluctant to include clinics in the CCO screening program. The reality is that screening should be performed in ambulatory clinics. Why should we take our healthiest patients into a hospital milieu for a routine outpatient procedure? The hospitals with the high cost per case structures should be reserved for patients with significant disease who require those facilities. 
There are a number of clinics that follow all of the published guidelines, including the CPSO IHF standards, the CCO quality guidelines and the Canadian Association of Gastroenterology screening (ie, interval) guidelines. They employ only well-trained nursing and technical staff, and only have gastroenterologists with active hospital privileges working there (the very doctors $\mathrm{CCO}$ uses in hospitals). While these gastroenterologists are not the only qualified physicians who perform colonoscopy, they are qualified nevertheless, and there will not be any concern about expertise and follow-up regardless of what is required after colonoscopy. I believe that if these centres are recognized and rewarded with cases by CCO (in Ontario), this designation will drive improved quality overall. Other clinics will either raise their standards or close through attrition. Hopefully, a credentialling process can be established using the existing guidelines so that we can ensure that only accredited facilities are performing out-of-hospital endoscopy. This accreditation will ensure access to quality colonoscopy in a safe, cost-efficient and appropriate nonhospital setting.

\section{REFERENCE}

1. Leddin D, Hunt R, Champion M, et al. Canadian Association of Gastroenterology and the Canadian Digestive Health Foundation: Guidelines on colon cancer screening. Can J

Gastroenterol 2004;18:93-9. 


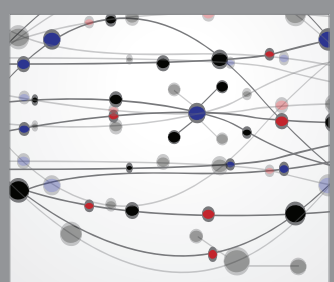

The Scientific World Journal
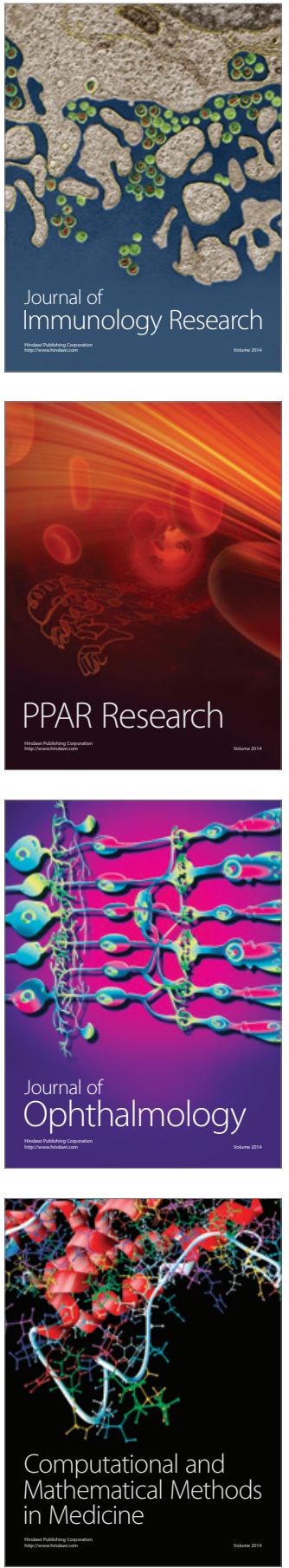

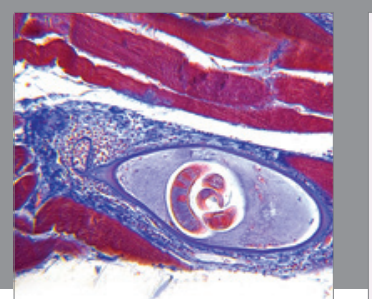

Gastroenterology Research and Practice

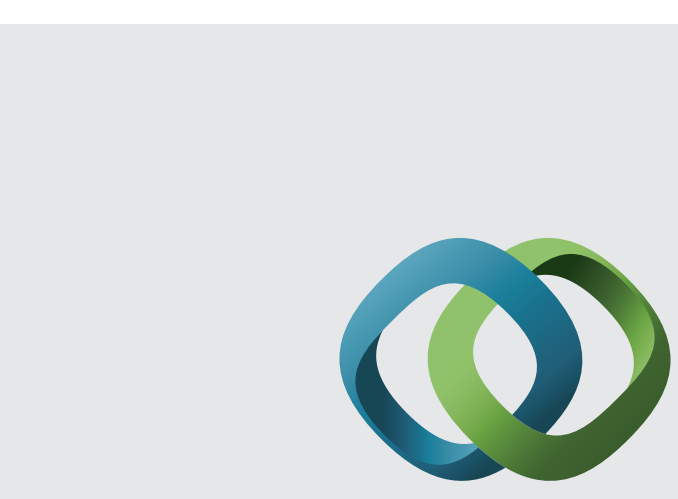

\section{Hindawi}

Submit your manuscripts at

http://www.hindawi.com
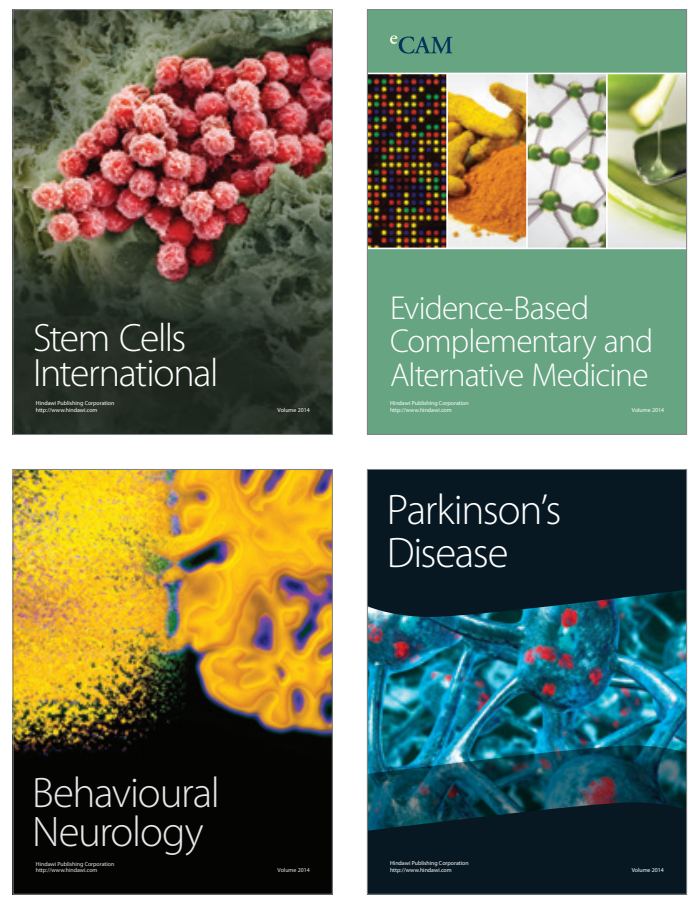
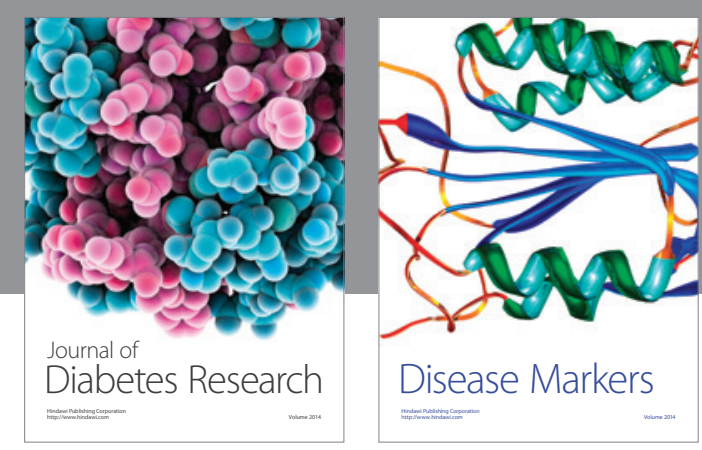

Disease Markers
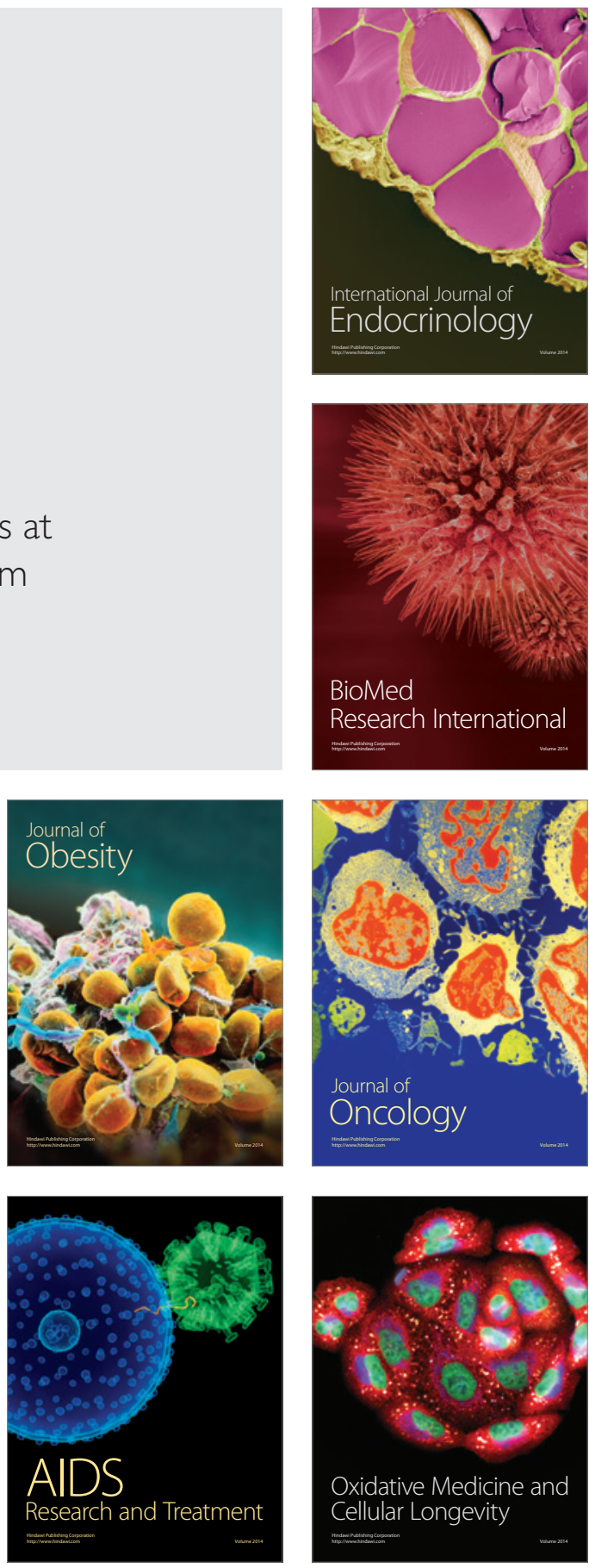